\title{
HOT SUBDWARFS IN BINARIES: MULTIWAVELENGTH OBSERVATIONS AND EVOLUTIONARY IMPLICATIONS
}

\author{
A. ULLA ${ }^{1,2}$, P. THEJLL ${ }^{1}$, S. VENNES ${ }^{3}$ \\ C. S. HANSEN-RUIZ ${ }^{4}$, J. L. RASILLA ${ }^{5}$ \\ 1. Niels Bohr Institute, Copenhagen, Denmark \\ 2. LAEFF-INTA, Madrid, Spain \\ 3. Center for Extreme Ultraviolet Astrophysics, Berkeley, USA \\ 4. Institute of Astronomy, Cambridge, UK \\ 5. Instituto de Astrofisica de Canarias, Spain
}

\begin{abstract}
We are looking for stellar companions to very hot helium-rich subluminous ( $\mathrm{sdO}$ ) stars by using infrared photometry, optical CCD imaging and spectroscopy. The work is complemented with a comprehensive bibliographic and space-mission databanks search for the objects in our sample (Thejll, Ulla \& MacDonald 1995). Companions can be used to indirectly measure the distance to the hot subdwarfs, and give clues about possible binary evolution scenarios. We have therefore gathered and analysed data for 57 hot subdwarfs in a search for IR excesses and companions. 43 of the stars are observed for the first time in the $J H K$ bands and 28 objects are found to have larger IR fluxes than expected from a single sdO.
\end{abstract}

\section{Introduction}

Hot helium-rich subdwarf stars (sdOs) are situated above the very blue end of the horizontal branch in the field and cluster populations. Beyond knowing that they are hot $(40000 \mathrm{~K}$ to $80000 \mathrm{~K})$ and helium-rich (photospheres are $10 \%$ to $100 \% \mathrm{He}$, by number), little is known about the evolutionary status, let alone origins, of these stars. The surface gravity, found in NLTE spectral analysis, is uncertainly known but tends to lie in the range $\log g=5.0 \ldots 6.5$. In the spectra, metals can be seen and these typically include $\mathrm{C}, \mathrm{N}$ and $\mathrm{Fe}$, but the abundances may not be original in that radiative levitation, gravitational settling and wind-driven mass loss are likely to alter the composition of the photospheres.

The spatial distribution of the sdOs is only tentatively known from the 469

A. Evans and J. H. Wood (eds.), Cataclysmic Variables and Related Objects, 469-470. (c) 1996 Kluwer Academic Publishers. Printed in the Netherlands. 
NLTE analysis of about 40 hot sdO stars coupled to the assumption of a mass near $0.5 \mathrm{M}_{\odot}$. Depending on the limiting magnitude of the observational material scale heights of at least $500 \mathrm{pc}$ for the population of sdOs can be surmised. The kinematics of hot sdOs are being investigated (Thejll \& Flynn, in preparation) for a large sample of stars. Previously, smaller samples of bright sdO kinematics have been analysed and divergent results have been found - but the results found are consistent with the sample limiting magnitudes, i.e. bright samples yield 'young' population memberships while the age increases with the limiting magnitude of the sample, thus indicating consistency with a large age for the population as a whole.

The binarity of the sdOs remains unknown, while that of their neighbors, the hot hydrogen-rich subdwarfs (sdBs), is observable at $50 \%$ and this implies a $100 \%$ binarity rate, as may be calculated from the assumed distribution over spectral classes of the companions. For the sdBs a scale height of $500 \mathrm{pc}$ has been reported (Saffer \& Liebert 1994). The sdB stars lie on the extended blue horizontal branch (EHB) and are currently identified with the helium-core burning stage of stellar evolution. Evolutionary tracks of such models lead from the EHB into the sdO region and thus suggest an evolutionary link that in turn requires similarity of scale heights, kinematics and rates of binarity between the sdBs and sdOs.

In a collaboration between photometrists, spectroscopists and modelers, and using available sources of information, we reported in this poster on a project designed to measure the rate of binarity of sdO stars. Beyond comparing the rate of binarity to that of the sdBs we can compare it to that of other potential progenitors and descendents such as the hydrogen-deficient $\mathrm{R}$ CrB stars and the helium-rich DB white dwarfs. It is also possible to use detected binary companions for derivation of atmospheric parameters of the hot subdwarf by measuring relative radii from multiwavelength photometry. A third interest lies in compiling a list of binary sdOs for separate investigations of the separation between the two stars; this has relevance for questions related to the possible co-evolution scenarios.

We have found that about $50 \%$ of the mainly s.ectroscopically selected sample of sdO stars are binary and we continue the analysis of these data.

\section{References}

Saffer, R.A., Liebert, J., 1994, in "White Dwarfs", Koester D. and Werner K. (eds.), Springer Verlag, p221

Thejll, P.A., Ulla A., MacDonald J., 1995, A\&A, 303, 773 